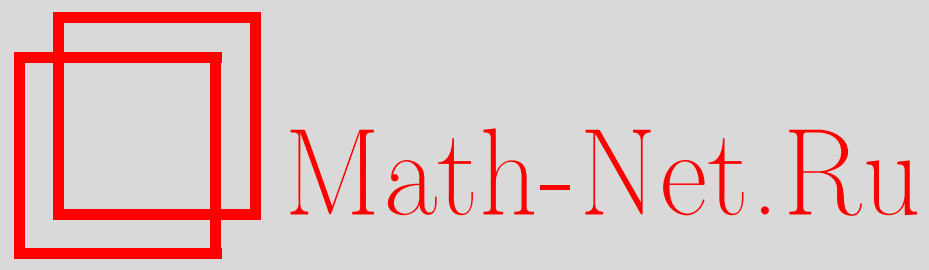

Р. И. Богданов, Факторизация диффеоморфизмов над фазовыми портретами векторных полей на плоскости, Функи. анализ и его прил., 1997, том 31, выпуск 2, 67-70

DOI: https://doi.org/10.4213/faa463

Использование Общероссийского математического портала MathNet.Ru подразумевает, что вы прочитали и согласны с пользовательским соглашением http://www . mathnet.ru/rus/agreement

Параметры загрузки:

IP : 54.209 .52 .79

26 апреля 2023 г., 06:56:57

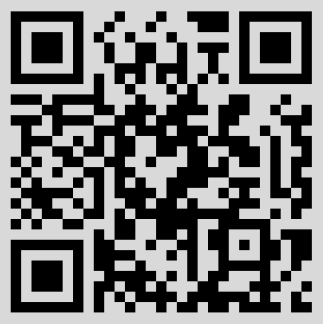




\title{
Факторизация диффеоморфизмов над фазовыми портретами векторных полей на плоскости ${ }^{*}$
}

\author{
(C) 1997. Р. И. БОГдАНОВ
}

В заметке изучаются инвариантные слоения диффеоморфизмов плоскости в окрестности неподвижной точки. Под слоением мы понимаем фазовый портрет бесконечно гладкого векторного поля на плоскости.

Основной результат представляет следующая

Теорема. Для диффеоморфизма плоскости в окрестности неподвижной точки общего положения (точнее, в конечномодальных случалх) существует двумерная $($ над $\mathbb{R})$ коммутативнал подалгебра в алгебре Ли гладких векторных полей, такал, что фазовые портреты ее векторных полей инвариантны относительно этого диффеоморфизма, причем диффеоморфизм или его квадрат включается в поток одного векторного поля подалгебры.

Особые точки диффеоморфизмов не общего положения (к которым неприменима сформулированная выше теорема) встречаются неустранимым образом в двупараметрических семействах диффеоморфизмов.

Ниже излагается схема доказательства приведенной теоремы (которая является следствием теоремы 3.3).

Автор выражает благодарность В. И. Арнольду, а также А. Г. Кушниренко и Ю. С. Ильяшенко за полезные обсуждения.

\section{1. Определения. Обозначения.}

1.1. ОБОзНАЧЕнИя. Через Vect (соответственно Diff) обозначим алгебру Ли (группу Ли) ростков в точке $(0) \in \mathbb{R}^{2}$ векторных полей (диффеоморфизмов) на плоскости класса $C^{\infty}$.

1.2. ОПРЕДЕЛЕНИЕ. Интегрируемой парой назовем дифФеоморфизм $g \in$ Diff и векторное поле $\mathbf{v} \in$ Vect, такие, что

$$
\operatorname{Ad}_{g} \mathbf{v}=\lambda \mathbf{v},
$$

где через $\operatorname{Ad}_{g}$ обозначено действие диффеоморфизма $g$ в присоединенном представлении группы Ли Diff на алгебре Ли Vect, а $\lambda$ - росток в точке $(0) \in \mathbb{R}^{2}$ скалярной функции на плоскости.

Обозначим интегрируемую пару через $g \vdash \mathbf{v}$.

1.3. ЗАмечание. Пара $g \in$ Diff и $\mathbf{v} \in$ Diff является интегрируемой парой, $g \vdash \mathbf{v}$, тогда и только тогда, когда фазовый портрет векторного поля $\mathbf{v}$ инвариантен относительно диффеоморфизма $g$.

\section{2. Кобордантные интегрируемые пары.}

2.1. ОПРЕДЕЛЕНИЕ. Две интегрируемые пары $g_{i} \vdash \mathbf{v}_{i}(i=0,1)$ назовем $\kappa o$ бордантными, если существует однопараметрическое семейство интегрируемых

* Работа выполнена при частичной поддержке фонда International Science Foundation и Российского правительства, грант М98300, и фонда РФФИ, грант 95-01-00229а. 
пар $g_{\varepsilon} \vdash \mathbf{v}_{\varepsilon}, \varepsilon \in[0,1]$, включающее в себя при $\varepsilon=0,1$ пары $g_{0} \vdash \mathbf{v}_{0}$ и $g_{1} \vdash \mathbf{v}_{1}$ соответственно.

2.2. Лемма. Пусть $g_{\varepsilon} \vdash \mathbf{v}_{\varepsilon}-$ росток в точке $\varepsilon=0$ однопараметрического семейства интегрируемых пар. Тогда имеет место соотношение

$$
L_{\mathbf{v}}(\boldsymbol{\xi} \wedge \mathbf{v})=\boldsymbol{\eta} \wedge \mathbf{v}-\left.\left(\frac{\operatorname{det} g}{\lambda^{2}} \boldsymbol{\eta} \wedge \mathbf{v}\right)\right|_{g^{-1}(\mathbf{x})},
$$

где $\mathbf{v}=\mathbf{v}_{0}, g=g_{0}, \boldsymbol{\xi}=\partial g_{\varepsilon} /\left.\partial \varepsilon\right|_{\varepsilon=0}, \boldsymbol{\eta}=\partial \mathbf{v}_{\varepsilon} /\left.\partial \varepsilon\right|_{\varepsilon=0}, \lambda-$ нормируюший множитель из (1), причем для некоторой функиии ${ }^{1}$ в выполнено условие

$$
\left.\frac{\partial \lambda_{\varepsilon}}{\partial \varepsilon}\right|_{\varepsilon=0}=F(\lambda, \mathbf{v}, \boldsymbol{\xi}, \boldsymbol{\eta}) \text {. }
$$

2.3. ТеоремА. Пусть семейство пар $g_{\varepsilon} \in$ Diff $u \mathbf{v}_{\varepsilon} \in$ Vect определено $в$ окрестности отрезка $\varepsilon \in[0,1]$ и при каждом $\varepsilon \in[0,1]$ выполнено соотношение (2), где $\lambda_{\varepsilon}$ - решение уравнения (3), причем пара $g_{0} \vdash \mathbf{v}_{0}$ интегрируемая. Тогда все пары этого семейства являются интегрируемыми, $g_{\varepsilon} \vdash \mathbf{v}_{\varepsilon}$, m. е. любые две пары этого семейства кобордантны между собой.

\section{3. Конечномодальные интегрируемые пары.}

3.1. ОБозНАЧЕНИЕ. Через $\Phi_{g \vdash \mathbf{v}}$ обозначим класс кобордантной эквивалентности интегрируемой пары $g \vdash \mathbf{v}$. Через $\pi \Phi_{g \vdash \mathbf{v}}$ обозначим проекцию класса кобордантной зквивалентности на пространство Diff.

3.2. ОПРЕДЕЛЕНИЕ. Интегрируемую пару $g \vdash \mathbf{v}$ назовем конечномодальной, если проекция $\pi \Phi_{g \vdash \mathbf{v}}$ имеет конечную коразмерность в пространстве Diff.

3.3. ТЕОРЕмА. Пусть конечномодальная интегрируемая пара $g \vdash \mathbf{v}$ maкова, что линеаризачия векторного поля $\mathbf{v}$ в точке $(0) \in \mathbb{R}^{2}$ не нильпотентна. Тогда конечномодальная интегрируемая пара $g \vdash \mathbf{v}$

(a) кобордантно эквивалентна одной и только одной из пар приведенной ниже табл. 1;

(b) диффеоморфизм этой кобордантно эквивалентной парьи или его квадрат включается в поток векторного поля исходной пары.

Заключение. В обзоре [3] приводятся результаты дискретизации по Эйлеру для двупараметрического семейства векторных полей. Это семейство векторных полей является версальной деформацией векторного поля, содержащего вырожденную особую точку. Вырождение заключается в обращении в нуль собственных чисел матрицы линеаризации векторного поля в особой точке (матрица эквивалентна жорданову блоку) (см. [1]). На иллюстрациях в [3] видны инвариантные слоения дискретного потока, связанного с конечноразностной аппроксимацией. Очевидно, что изложенные выше результаты позволяют проводить более обоснованно такие расчеты.

Слово «факторизация» в названии заметки становится ясным при изучении табл. 1. Уместно заметить при этом, что в окрестности неособой точки векторного поля интегрируемой пары диффеоморфизм из этой пары является полупрямым произведением. Прямой сомножитель отвечает действию диффеоморфизма на локальной алгебре первых интегралов векторного поля пары, которая инвариантна относительно названного действия.

${ }^{1}$ Мы не указываем явно выражение для $F$, потому что оно сложное и необходимо лишь при изложении полного доказательства результатов. 
Таблица 1. Нормальные формы интегрируемых пар с точностью до кобордизма пар

\begin{tabular}{|c|c|c|c|c|c|}
\hline Имя & $g: \mathbf{x}(\bigvee \mathbf{z}) \mapsto$ & $\xi$ & $\eta$ & $\mathbf{v}$ & Комментарии \\
\hline & $\left(\begin{array}{ll}e^{s \Delta t} & x_{1} \\
e^{\lambda \Delta t} & x_{2}\end{array}\right)$ & $x_{1} \partial_{1}$ & $x_{2} \partial_{2}$ & $s \boldsymbol{\xi}+\lambda \boldsymbol{\eta}$ & $\left(\begin{array}{c}s \\
\lambda\end{array}\right)-\begin{array}{l}\text { нерезонансный } \\
\text { вектор }\end{array}$ \\
\hline & $e^{s \Delta t}\left(\begin{array}{c}x_{1}+\Delta t x_{2} \\
x_{2}\end{array}\right)$ & $\mathbf{e}$ & $x_{2} \partial_{1}$ & $s \boldsymbol{\xi}+\boldsymbol{\eta}$ & $\begin{array}{c}\text { жорданова клетка, } \\
\Delta t \neq 0\end{array}$ \\
\hline & $e^{c \Delta t} e^{-i \Delta t} \mathbf{z}$ & $\mathbf{e}$ & $\operatorname{Id}\left(\frac{H}{2}\right)$ & $c \boldsymbol{\xi}+\boldsymbol{\eta}$ & $\begin{array}{c}H=\mathbf{z} \overline{\mathbf{z}}=x_{1}^{2}+x_{2}^{2}, \\
c \neq 0\end{array}$ \\
\hline$A_{j}^{ \pm}$ & $\Theta^{-1}\left(\begin{array}{c}{\left[e^{\Delta t} \Theta^{-c s}\right]^{m} x_{1}} \\
{\left[e^{\Delta t} \Theta^{-c s}\right]^{n} x_{2}}\end{array}\right)$ & $\Lambda \mathbf{x}$ & $-c H^{j} \boldsymbol{\xi}+s H^{j} \mathbf{e}$ & $\boldsymbol{\xi}+\boldsymbol{\eta}$ & $\begin{array}{c}\Lambda \mathbf{x}=m x_{1} \partial_{1}-n x_{2} \partial_{2} \\
H=x_{1}^{n} x_{2}^{m}\end{array}$ \\
\hline$C_{j}^{ \pm}$ & $e^{-i \Delta t} \Theta^{c s / 2} \mathbf{z}$ & $\operatorname{Id}\left(\frac{H}{2}\right)$ & $-c H^{j} \boldsymbol{\xi}+s H^{j} \mathbf{e}$ & $\boldsymbol{\xi}+\boldsymbol{\eta}$ & $\begin{array}{l}H=\mathbf{z} \overline{\mathbf{z}}, \\
\Delta t-\begin{array}{l}\text { нерезонансная } \\
\text { величина }\end{array}\end{array}$ \\
\hline$B_{j}^{ \pm}$ & $\left(\begin{array}{c}\Theta^{-1} x_{1} \\
e^{p s \Delta t} \Theta^{-c s} x_{2}\end{array}\right)$ & $x_{2} \partial_{2}$ & $-c x_{1}^{j} \boldsymbol{\xi}+s x_{1}^{j+1} \partial_{1}$ & $p \boldsymbol{\xi}+\boldsymbol{\eta}$ & $H=x_{1}, \quad p= \pm 1$ \\
\hline$D_{j}^{ \pm}$ & $\left(\begin{array}{c}e^{-s \Delta t} x_{1} \\
e^{s j \Delta t}\left(x_{2}+p \Delta t x_{1}^{j+1}\right)\end{array}\right)$ & $\mathbf{w}$ & $x_{1}^{j} \partial_{2}$ & $s \mathbf{w}+p \boldsymbol{\eta}$ & $\begin{array}{c}\mathbf{w}=x_{1} \partial_{1}+j x_{2} \partial_{2} \\
p=0, \pm 1\end{array}$ \\
\hline
\end{tabular}

Здесь $g$ является фазовым потоком $g_{\Delta t}(\mathbf{x})$ векторного поля $\mathbf{v} \in \mathbb{R}\langle\boldsymbol{\xi}, \boldsymbol{\eta}\rangle, \Delta t \neq 0$, $\Delta t \in \mathbb{R},[\boldsymbol{\xi}, \boldsymbol{\eta}]=0, s= \pm 1, j \in \mathbb{Z}^{+}, c, \lambda \in \mathbb{R}, \mathbf{z}=x_{1}+i x_{2} \in \mathbb{C}, m, n \in \mathbb{Z}^{+},(m, n)=1$, $\Theta=\left(1-j s H^{j} \Delta t\right)^{1 / j}, \mathbf{e}=x_{1} \partial_{1}+x_{2} \partial_{2}, \operatorname{Id} H=\left(\partial H / \partial x_{2}\right) \partial_{1}-\left(\partial H / \partial x_{1}\right) \partial_{2}$.

Библиографическое дополнение. Теорема, сформулированная в начале заметки, восходит к работе Babbage Ch. An essay towards the calculus of functions. Philosophical Transactions of the Royal Society of London, 105, 389-423 (1815). В линейном случае (первые три строки табл. 1) она тривиальна. Со времен работ А. Пуанкаре известно, что в топологическом смысле диффеоморфизмом общего положения в пространстве диффеоморфизмов в окрестности стационара являются линейные диффеоморфизмы (см. [1] и библиографию там же). К сожалению, в численных приложениях (см., например, [3]) топологическое общее положение не гарантирует устойчивости численных алгоритмов. Диффеоморфизмы Аносова (см. [2]) показали, что отыскание инвариантных слоений по существу является неустойчивой задачей в топологически типичных случаях.

Таблица, приведенная в заметке, проясняет смысл устойчивых численных методов получения инвариантных слоений локально в окрестности стационара общего положения. Здесь общность положения понимается не в топологическом, а даже в естественном метрическом смысле.

Метод, предложенный в заметке, по сути подхода ближе идеям С. Ли (см. Lie Groups: History, Frontiers and Applications, Vol. 1, Sophus Lie's 1880 Transformation Group Paper. Transl. by M. Ackermann, Comm. by R. Hermann, Math. Sci. Press, Brookline, Massachusetts 02146 (1975).) С. Ли знал свойства ядра оператора, выписанного в левой части гомологического уравнения (2), и его связь с алгеброй Галуа фазового портрета векторного поля. Гомологические уравнения в бесконечномерном случае были недоступны как С. Ли, так и Галуа и в текущем столетии не использовались в полной мере в качественной теории динамических систем и теории их бифуркаций (за исключением метода, восходящего к работам Пуанкаре прошлого столетия (см. [1])).

В настоящее время анонсирован результат (Bogdanov R. I. Weakly Dissipative Theory by Kolmogorov-Arnold-Moser. Abstracts of Int. Conf. on Contemporary Problems in Theory of Dynamical Systems, 1-6 July, 1996, Nizhny Novgorod University), показывающий, что предложенные методы позволяют в примере [3] исследовать периодические траектории вплоть до периодов $\sim 10^{5}$ (подробнее см. Богданов Р. И. Приложения слабо-диссипативной теории Колмогорова-Арнольда-Мозера. Препринт НИИЯФ МГУ 96-22/429, М., 1996).

Безусловно, заметка обязана главе об эквивариантных векторных полях в книге В. И. Арнольда [1]. 


\title{
ЛитеРАтУРА
}

1. Арнольд В. И. Дополнительные главы теории обыкновенных дифференциальных уравнений. Наука, М., 1978. 2. Аносов Д. В. Геодезические потоки на замкнутых римановых многообразиях отрицательной кривизны. Труды МИАН им. В. А. Стеклова, 90, 1967. 3. Arrowsmith D. K., Cartwright J. H. E., Lansbury A. N., Place C. M. Internat. J. Bifurcation and Chaos, 3, No. 4, 803-842 (1993). 4. Богданов Р. И. Локальная орбитальная эквивалентность векторных полей на плоскости. Изд-во МГУ, 1993. 5. Ильяшенко Ю. С., Яковенко С. Ю.УМН, 46, №1, 3-40 (1991).

Московский государственный университет им. М. В. Ломоносова, Поступило в редакцию Институт ядерной физики

7 июня 1995 г.

УДК $517.9+513.015 .7$

\section{Решения уравнения Янга и алгебраические кривые рода, большего 1}

\author{
(c) 1997. В. И. ДРАГОвИч
}

В конце восьмидесятых годов Бакстер и др. (см. [1]), исследуя так называемую модель Поттса, нашли решения уравнения Янга

$$
R L L^{\prime}=L^{\prime} L R
$$

и уравнения Янга-Бакстера

$$
R^{12}\left(\Theta_{1}\right) R^{13}\left(\Theta_{2}\right) R^{23}\left(\Theta_{3}\right)=R^{23}\left(\Theta_{3}\right) R^{13}\left(\Theta_{2}\right) R^{12}\left(\Theta_{1}\right)
$$

со спектральным параметром, который параметризован кривой рода, большего 1. Доказано, что такие решения не удовлетворяют условию $\Theta_{3}=\Theta_{2}-\Theta_{1}$, и поэтому казалось, что они никак не связаны с уже известными решениями.

Но В. В. Бажанов и Ю. Г. Строганов нашли следуюшую связь (см. [2]). Они решали уравнение $(1)$, где в качестве $(4 \times 4)$-матрицы $R$ была взята $R$-матрица шестивершинной модели $R=R_{X X Z}$, и искали неизвестные $(2 n \times 2 n)$-матрицы $L$ и $L^{\prime}$. (Дополнительно был задан вид матриц $L$ и $L^{\prime}: L(x)=x L_{+}+x^{-1} L_{-}$.) Затем они рассматривали уравнение (1) с полученными таким образом матрицами $L$ и $L^{\prime}$ как уравнение с неизвестной $\left(n^{2} \times n^{2}\right)$-матрицей $R$. Выяснилось, что в качестве решения появляется именно $R$-матрица модели Поттса.

В этой работе мы исследуем общие решения уравнения (1), где $R$ - матрица размера $n^{2} \times n^{2}$, а $L$ и $L^{\prime}$ - матрицы размера $2 n \times 2 n$. Полученные матрицы будут иметь спектральные кривые рода, большего 1 , с нетривиальной группой автоморфизмов. Напомним, что в случае модели Поттса появляется кривая Ферма, которая, как известно, имеет нетривиальную группу автоморфизмов.

1. Спектральные кривые. Алгебро-геометрическая параметризация четномерных матриц, основанная на понятии вакуумных векторов и связанных с 